\title{
Genome Sequence Resource for Stagonosporopsis cucurbitacearum, a Cause of Gummy Stem Blight Disease of Watermelon
}

\author{
Fahao Wang, Jiahui Qi, Miao Tian, Yizhou Gao, Xiaohui Xiong, Jiajing Wang, Fengming \\ Song, ${ }^{\dagger}$ and Dayong $\mathrm{Li}^{\dagger}$
}

Key Laboratory of Crop Diseases and Insect Pests of Ministry of Agriculture, Institute of Biotechnology, Zhejiang University, Hangzhou 310058, P. R. China

\section{Abstract}

Gummy stem blight (GSB), which is caused by three related species of Stagonosporopsis, is a worldwide devastating disease of cucurbit crops including watermelon. Previously S. cucurbitacearum was reported to be the major fungal cause of watermelon GSB in Southern China, where it causes a significant decrease in watermelon yield. Here, we present the draft whole genome sequence, gene prediction and annotation of S. cucurbitacearum strain DBTL4, isolated from diseased watermelon plants. To our knowledge, this is the first publicly available genome sequence of this species, and knowledge of this genome sequence will help further understand the pathogenic mechanism of S. cucurbitacearum to cucurbit plants.

\section{Genome Announcement}

Watermelon (Citrullus lanatus (Thunb.) Matsum. \& Nakai) is an economically important fruit in the world and the total annual yield in 2019 was over 100 million tons (FAO Stat 2019). Gummy stem blight (GSB), caused by the three morphologically indistinguishable but genetically distinct species Stagonosporopsis cucurbitacearum, S. citrulli, and S. caricae, is a very common disease on cucurbitaceous crops, including watermelon, cantaloupe, brandy melon, pumpkin, muskmelon, and cucumber, throughout the world, especially in regions with humid and warm weather (Keinath 2011; Keinath et al. 1995; Li et al. 2015; Stewart et al. 2015; van Steekelenburg 1983). The three Stagonosporopsis spp. are pathogenic to cucurbits but S. caricae also causes disease on papaya (Carica papaya). Although sexual and asexual reproductive structures, including ascospores in pseudothecia and conidia in pycnidia, respectively, can appear on lesions throughout the growing season, they play different roles during the infections. Beyond that, the disease can cause watermelon leaves and vines to die and fruit to rot, which leads to loss of watermelon fruit in storage and transportation (Gusmini et al. 2017; Keinath et al. 1995). In areas with severe GSB disease, the yield losses of watermelon were estimated up to $100 \%$ (Keinath 2014; St. Amand and Wehner 1995). Although S. cucurbitacearum is prevalent and causes severe damage to watermelon and other cucurbit crops in several countries, including the United States and China

\footnotetext{
${ }^{\dagger}$ Corresponding authors: D. Li; dyli@zju.edu.cn and F. Song; fmsong@ zju.edu.cn

*The $e$-Xtra logo stands for "electronic extra" and indicates that a supplementary table is published online.
}

The author(s) declare no conflict of interest.

\section{$e-X \operatorname{tra}^{*}$}

\section{Funding}

This work was supported by the National Key Research and

Development Program of China (grant number 2018YFD0201300) and the Earmarked Fund for Modern AgroIndustry Technology Research System (CARS-26-11). 
Table 1. Summary statistics of the Stagonosporopsis cucurbitacearum DBTL4 genome

\begin{tabular}{ll} 
Variables $^{\text {a }}$ & \multicolumn{1}{c}{ Statistics } \\
Sequence coverage & $125.86 \times$ \\
Genome size $(\mathrm{Mb})$ & 34.08 \\
Number of scaffolds & 20 \\
Scaffold $N_{50}$ size (bp) & $1,997,632$ \\
Maximum scaffold size (bp) & $2,854,808$ \\
$\mathrm{~N}_{50}$ & 18,813 \\
$\mathrm{GC}$ content (\%) & 50.76 \\
Number of predicted genes & 10,748 \\
Number of predicted secreted proteins & 812 \\
BUSCO completeness (\%) & 97.93 \\
Genome accession & JAFEKD010000000 \\
\hline
\end{tabular}

${ }^{a}$ BUSCO = Benchmarking Universal Single-Copy Ortholog.

(Everts 1999; Li et al. 2015), there is not fully annotated, publicly available information on the genome of $S$. cucurbitacearum. Therefore, genome sequencing and annotation are important steps toward understanding the pathogenic mechanism of S. cucurbitacearum.

In this study, the $S$. cucurbitacearum strain DBTL4 was isolated from infected watermelon leaves from Tonglu City, Zhejiang, China. Based on morphological characteristics and multigene phylogenetic analyses of the internal transcribed spacer (Zhao et al. 2018, 2020), DBTL4 was identified as $S$. cucurbitacearum. The genomic DNA was extracted and purified from mycelium using a Genomic-tip 100/G extraction kit (Qiagen). The Thermo Scientific NanoDrop 2000 and Qubit systems were utilized to evaluate DNA purity and content, and $0.35 \%$ agarose gel electrophoresis was carried out to test the DNA integrity. We recycled large segments of DNA using the BluePippin system and prepared a library using the ligation sequencing kit SQK-LSK109 (Oxford Nanopore Technologies [ONT], Oxford, U.K.) and New England Biolabs (NEB) Next FFPE DNA repair mix kit.

The high-quality library was sequenced on the ONT PromethION platform (ONT) using the ligation sequencing kit (SQKLSK109) and the flow cell priming kit (EXP-FLP002). Subreads that were of low quality and too short were removed, and the remaining subreads were used for genome assembly. Due to a high error rate of Nanopore reads, we first corrected reads by the error correction module of Canu v1.7.1 software (Koren et al. 2017). All settings were the default. Next, the corrected reads were independently assembled with wtdbg v2.2 (Ruan and Li 2020). To obtain more accurate genome data, the sequencing library was prepared using the Nextera DNA Flex library preparation kit (Illumina, San Diego, CA, U.S.A.) according to the manufacturer's instructions and sequenced on an Illumina Nova-Seq 6000 platform. Illumina short reads were used to correct the sequencing errors generated by Oxford Nanopore PromethION sequencing, using Pilon software (Walker et al. 2014) with default settings.

Thereafter, the completeness of the genome assembly was assessed using the Benchmarking Universal Single-Copy Ortholog (BUSCO) v3.0 program (Simão et al. 2015). This analysis indicated that the completeness of the DBTL4 genome assembly was $97.93 \%$ and there were 284 complete BUSCOs, 3 missing BUSCOs, 3 fragmented BUSCOs, and 290 total lineage BUSCOs.

In total, $4.63 \mathrm{~Gb}$ of genome sequence was obtained from 320,252 subreads. After a correction of 1,976,201 bp (substitution $=415,435 \mathrm{bp}$, deletion $=660,263 \mathrm{bp}$, and insertion $=$ $900,503 \mathrm{bp}$ ) on the preassembled genome, the final genome had a total assembly length of $34,085,764 \mathrm{bp}$, which is smaller than that of $S$. cucurbitacearum strains sequenced with PacBio RS II sequencing (Zhao et al. 2020) and contained 20 scaffolds, with an $\mathrm{N}_{50}$ value of $1,997,632$ bp and a GC content of $50.76 \%$. The maximum scaffold size was $2,854,808$ bp and other characteristics of this genome are summarized in Table 1. The fungal genome repeat sequence database was first constructed based on the principle of structure prediction and $a b$ initio prediction with the help of four software programs; namely, LTR_FINDER v1.05 (Xu and Wang 2007), MITE-Hunter (Han and Wessler 2010), RepeatScout v1.0.5 (Price et al. 2005), and PILER-DF v2.4 (Edgar and Myers 2005). The constructed database was then classified using PASTEClassifier (Wicker et al. 2007) and merged with the Repbase 
(Jurka et al. 2005) database to generate the final repeat sequence database. The repeat sequences of the fungus were predicted based on the constructed repeat sequence database, using RepeatMasker v4.0.6 software (Chen 2004), and a repetitive sequence of $3,477,720$ bp was obtained, with a repetitive sequence ratio of $10.20 \%$.

To better predict protein-coding genes, we obtained RNA-sequencing (RNA-seq) data for strain DBTL4 using the Nova-Seq 6000 system. The total RNA was extracted from mycelium samples using RNAprep Pure Plant Kit (Tiangen). In total, $1 \mu \mathrm{g}$ of RNA per sample was used as input material for the RNA sample preparations. Sequencing libraries were generated using NEBNext Ultra RNA Library Prep Kit for Illumina (NEB) following the manufacturer's recommendations and index codes were added to attribute sequences to each sample. The RNA-seq data were submitted to GenBank under accession numbers SRR13528758, SRR13528759, and SRR13528760. We obtained 27,321,963 read pairs with a final total length of 8,184,715,274 bp. The percentages of Q20 and Q30 bases were 97.33 and $92.65 \%$, respectively. The genome was annotated based on three different prediction strategies: ab initio prediction, RNA-seq-based prediction, and homology-based prediction. For RNA-seq-based prediction, the RNA-seq reads were aligned against our genome using Hisat2 v2.0.4 and Stringtie v1.2.3 (Pertea et al. 2016) to generate spliced alignments. Next, the RNA-seq reads were assembled using Trinity software (v2.1.1). Subsequently, the assembled transcripts were used for gene model prediction with TransDecoder v2.0 and PASA v2.4.1 (Campbell et al. 2006), separately. The ab initio gene predictions were performed with Augustus v2.4 (Stanke and Waack 2003), Genscan (Burge and Karlin 1997), and GlimmerHMM v3.0.4 (Majoros et al. 2004), with parameters trained on the PASA predictions. For the homology-based prediction, sequenced genomes of the Didymella genus (Didymella exigua) from NCBI were used to predict gene models with GeMoMa v1.3.1 (Keilwagen et al. 2016). Finally, RNA-seq-based gene models, ab-initio-based gene models, and homology-based gene models were combined using EVidenceModeler (Haas et al. 2008) to integrate a final gene set. Default settings were used for all the programs.

In total, 10,748 protein-coding genes were predicted. By analyzing the predicted protein sequences, 812 secreted proteins (referring to proteins containing an $\mathrm{N}$-terminal signal peptide cleavage site and with no transmembrane domain after the region signal peptide cleavage site) were predicted using SignalP 5.0 (Petersen et al. 2011) and TMHMM 2.0 (Krogh et al. 2001) software. In the secretome, 113 proteins were identified as potential effectors using EffectorP 5.0 software, which may play vital roles in inactivating the host defense system (Sperschneider et al. 2018). Putative carbohydrate-active enzymes (CAZymes) were predicted using dbCAN (Yin et al. 2012). We identified 635 CAZymes, including 100 auxiliary activity family proteins, 273 glycoside hydrolases, 95 glycosyltransferases, 36 polysaccharide lyases, 122 carbohydrate esterases, and 59 noncatalytic carbohydrate-binding modules. Moreover, 2,273 transmembrane proteins were predicted by TMHMM software (Krogh et al. 2001), and 143 transport proteins and 3,101 pathogen-host interaction (PHI) proteins were identified in searches of the transporter classification and PHI databases, respectively (Saier et al. 2006; Winnenburg et al. 2006, 2008).

In addition, we compared the strain DBTL4 assembly with the recently published $S$. cucurbitacearum strain zq-1 assembly isolated from Northeast China (Zhao et al. 2020). Based on these comparisons (Supplementary Table S1), the DBTL4 assembly is markedly better than the assembly of zq-1 in terms of the number of scaffolds and scaffold $\mathrm{N}_{50}$. Our results provided a high-quality genome annotation reference of $S$. cucurbitacearum and an advanced research method on the genome sequencing of plant pathogens.

Although another whole-genome sequencing and genome assembly of S. cucurbitacearum has been reported recently (Zhao et al. 2020), the genomic data are not publicly available. The genome described here provides the first publicly available genomic data for S. cucurbitacearum. This reference genome and annotation will provide insights into the pathogenic mechanism of $S$. cucurbitacearum and its molecular interactions with host plants. The Nanopore read data generated in this study have been submitted to GenBank under BioProject (PRJNA694739), BioSample (SAMN17574046), and Sequence Read Archive (SRR13528369). The contig assembly of this draft genome has been deposited in GenBank under the accession number JAFEKD000000000. The version described in this article is version JAFEKD010000000. The genome annotation files (CDS, gff, and proteins) are available in Figshare.

Vol. 34, No. 8, 2021 / 979 


\section{Acknowledgments}

We thank C. Jiao from the Institute of Biotechnology, Zhejiang University and J. Yang from the Department of Horticulture, Zhejiang University for helpful suggestions for this article.

\section{Author-Recommended Internet Resources}

figshare: https://figshare.com/articles/online_resource/Stagonosporopsis_cucurbitacearum_CDS/13980500

TransDecoder v2.0: https://github.com/TransDecoder

\section{Literature Cited}

Burge, C., and Karlin, S. 1997. Prediction of complete gene structures in human genomic DNA. J. Mol. Biol. 268:78-94.

Campbell, M. A., Haas, B. J., Hamilton, J. P., Mount, S. M., and Buell, C. R. 2006. Comprehensive analysis of alternative splicing in rice and comparative analyses with Arabidopsis. BMC Genomics 7:327.

Chen, N. 2004. Using RepeatMasker to identify repetitive elements in genomic sequences. Curr. Protoc. Bioinf. 5:4.10.1-4.10.14.

Edgar, R. C., and Myers, E. W. 2005. PILER: Identification and classification of genomic repeats. Bioinformatics 21:i152-i158.

Everts, K. L. 1999. First report of benomyl resistance in Didymella bryoniae in Delaware and Maryland. Plant Dis. 83:304.

FAO Stat. 2019. FAO Statistics. http://www.fao.org/faostat/zh/\#data/QC

Gusmini, G., Rivera-Burgos, L. A., and Wehner, T. C. 2017. Inheritance of resistance to gummy stem blight in watermelon. HortScience 52:1477-1482.

Haas, B. J., Salzberg, S. L., Zhu, W., Pertea, M., Allen, J. E., Orvis, J., White, O., Buell, C. R., and Wortman, J. R. 2008. Automated eukaryotic gene structure annotation using EVidenceModeler and the Program to Assemble Spliced Alignments. Genome Biol. 9:R7.

Han, Y., and Wessler, S. R. 2010. MITE-Hunter: A program for discovering miniature inverted-repeat transposable elements from genomic sequences. Nucleic Acids Res. 38:e199.

Jurka, J., Kapitonov, V. V., Pavlicek, A., Klonowski, P., Kohany, O., and Walichiewicz, J. 2005. Repbase Update, a database of eukaryotic repetitive elements. Cytogenet. Genome Res. 110:462-467.

Keilwagen, J., Wenk, M., Erickson, J. L., Schattat, M. H., Grau, J., and Hartung, F. 2016. Using intron position conservation for homology-based gene prediction. Nucleic Acids Res. 44:e89.

Keinath, A. P. 2011. From native plants in Central Europe to cultivated crops worldwide: The emergence of Didymella bryoniae as a cucurbit pathogen. HortScience 46:532-535.

Keinath, A. P. 2014. Differential susceptibility of nine cucurbit species to the foliar blight and crown canker phases of gummy stem blight. Plant Dis. 98 : 247-254.

Keinath, A. P., Farnham, M. W., and Zitter, T. A. 1995. Morphological, pathological, and genetic differentiation of Didymella bryoniae and Phoma spp. isolated from Cucurbits. Phytopathology 85:364-369.

Koren, S., Walenz, B. P., Berlin, K., Miller, J. R., Bergman, N. H., and Phillippy, A. M. 2017. Canu: Scalable and accurate long-read assembly via adaptive k-mer weighting and repeat separation. Genome Res. 27:722-736.

Krogh, A., Larsson, B., von Heijne, G., and Sonnhammer, E. L. 2001. Predicting transmembrane protein topology with a hidden Markov model: Application to complete genomes. J. Mol. Biol. 305:567-580.

Li, P. F., Ren, R. S., Yao, X. F., Xu, J. H., Babu, B., Paret, M. L., and Yang, X. P. 2015. Identification and characterization of the causal agent of gummy stem blight from muskmelon and watermelon in East China. J. Phytopathol. 163:314-319.

Majoros, W. H., Pertea, M., and Salzberg, S. L. 2004. TigrScan and GlimmerHMM: Two open source ab initio eukaryotic gene-finders. Bioinformatics 20:2878-2879.

Pertea, M., Kim, D., Pertea, G. M., Leek, J. T., and Salzberg, S. L. 2016. Transcriptlevel expression analysis of RNA-seq experiments with HISAT, StringTie and Ballgown. Nat. Protoc. 11:1650-1667.
Petersen, T. N., Brunak, S., von Heijne, G., and Nielsen, H. 2011. SignalP 4.0: Discriminating signal peptides from transmembrane regions. Nat. Methods 8 : 785-786.

Price, A. L., Jones, N. C., and Pevzner, P. A. 2005. De novo identification of repeat families in large genomes. Bioinformatics 21:i351-i358.

Ruan, J., and Li, H. 2020. Fast and accurate long-read assembly with wtdbg2. Nat. Methods 17:155-158.

Saier, M. H., Jr., Tran, C. V., and Barabote, R. D. 2006. TCDB: The Transporter Classification Database for membrane transport protein analyses and information. Nucleic Acids Res. 34:D181-D186.

Simão, F. A., Waterhouse, R. M., loannidis, P., Kriventseva, E. V., and Zdobnov, E. M. 2015. BUSCO: Assessing genome assembly and annotation completeness with single-copy orthologs. Bioinformatics 31:3210-3212.

Sperschneider, J., Dodds, P. N., Gardiner, D. M., Singh, K. B., and Taylor, J. M. 2018. Improved prediction of fungal effector proteins from secretomes with EffectorP 2.0. Mol. Plant Pathol. 19:2094-2110.

St. Amand, P. C., and Wehner, T. C. 1995. Greenhouse, detached-leaf, and field testing methods to determine cucumber resistance to gummy stem blight. J. Am. Soc. Hortic. Sci. 120:673-680. enc.

Stanke, M., and Waack, S. 2003. Gene prediction with a hidden Markov model and a new intron submodel. Bioinformatics 19:ii215-ii225.

Stewart, J. E., Turner, A. N., and Brewer, M. T. 2015. Evolutionary history and variation in host range of three Stagonosporopsis species causing gummy stem blight of cucurbits. Fungal Biol. 119:370-382.

van Steekelenburg, N. A. M. 1983. Epidemiological aspects of Didymella bryoniae, the cause of stem and fruit rot of cucumber. Neth. J. Plant Pathol. 89:75-86.

Walker, B. J., Abeel, T., Shea, T., Priest, M., Abouelliel, A., Sakthikumar, S., Cuomo, C. A., Zeng, Q., Wortman, J., Young, S. K., and Earl, A. M. 2014. Pilon: An integrated tool for comprehensive microbial variant detection and genome assembly improvement. PLoS One 9:e112963.

Wicker, T., Sabot, F., Hua-Van, A., Bennetzen, J. L., Capy, P., Chalhoub, B., Flavell, A., Leroy, P., Morgante, M., Panaud, O., Paux, E., SanMiguel, P., and Schulman, A. H. 2007. A unified classification system for eukaryotic transposable elements. Nat. Rev. Genet. 8:973-982.

Winnenburg, R., Baldwin, T. K., Urban, M., Rawlings, C., Köhler, J., and Hammond-Kosack, K. E. 2006. PHI-base: A new database for pathogen host interactions. Nucleic Acids Res. 34:D459-D464.

Winnenburg, R., Urban, M., Beacham, A., Baldwin, T. K., Holland, S., Lindeberg, M., Hansen, H., Rawlings, C., Hammond-Kosack, K. E., and Köhler, J. 2008. PHI-base update: Additions to the pathogen host interaction database. Nucleic Acids Res. 36:D572-D576.

$\mathrm{Xu}, \mathrm{Z}$., and Wang, H. 2007. LTR_FINDER: An efficient tool for the prediction of full-length LTR retrotransposons. Nucleic Acids Res. 35:W265-W268.

Yin, Y., Mao, X., Yang, J., Chen, X., Mao, F., and Xu, Y. 2012. dbCAN: A web resource for automated carbohydrate-active enzyme annotation. Nucleic Acids Res. 40:W445-W451.

Zhao, Q., Wu, J., Zhang, L., Xu, L., Yan, C., and Gong, Z. 2018. Identification and characterization of Cucurbita gummy stem blight fungi in Northeast China. J. Phytopathol. 166:305-313.

Zhao, Q., Wu, J., Zhang, L., Yan, C., Jiang, S., Li, Z., Sun, D., Lai, Y., and Gong, Z. 2020. Genome-scale analyses and characteristics of putative pathogenicity genes of Stagonosporopsis cucurbitacearum, a pumpkin gummy stem blight fungus. Sci. Rep. 10:18065. 